\title{
Thromboelastography: Current Applications, Future Directions
}

\section{Linda M. Trapani}

The Brooklyn Hospital Center, Brooklyn, USA.

Email: ltrapani@alum.wellesley.edu

Received October 20 ${ }^{\text {th }}, 2012$; revised November $25^{\text {th }}, 2012$; accepted December $20^{\text {th }}, 2012$

\begin{abstract}
Analyzing coagulability often hinges on patient surveillance using prothrombin time (PT) or international normalized ratio (INR) and activated partial thromboplastin time (aPTT) to monitor the extrinsic and intrinsic coagulation pathways, respectively A more complete assessment, however, can often be obtained using thromboelastography (TEG), a coagulation assay that evaluates the efficiency of clot formation, as well as the viscoelastic properties of the clot. Developed by Dr. Helmut Hartert in 1948 at the University of Heidelberg, it provides information regarding hemostasis as a dynamic process [1,2]. Here, the TEG technique will be described, as well as its current applications and future directions for its use.
\end{abstract}

Keywords: Thromboelastography; Rotational Thromboelastometry; Cardiopulmonary Bypass; Coagulation Index; Adenosine Diphosphate; Prothrombin Time; Partial Thromboplastin Time; International Normalized Ratio

\section{Introduction}

The coagulation cascade is a highly regulated process relying on both the enzymatic activity of clotting factors as well as proper platelet structure and function. Several factors influence the function of platelets, including adenosine diphosphate (ADP), epinephrine, collagen and thrombin [2]. Routine coagulation assays, such as aPTT and PT, are not performed on whole blood; rather, these tests are performed only on plasma. Thus, the contribution of platelets to clot strength and formation is not considered. While PT and aPTT are often suitable tests for monitoring disorders of blood coagulation and medications used to treat these disorders, they provide an overlysimplistic view of global coagulation characteristics, particularly in critically ill patients. The impact of platelet function- or lack thereof-on clotting homeostasis can be crucial for intraoperative decision-making, especially regarding the need to transfuse blood products and clotting factors.

\section{Method}

\subsection{Performing the Technique}

In classical TEG, a small cuvette of blood is slowly rotated through an arc of about 4.75 degrees, six times per minute, to imitate sluggish venous flow and activate coagulation. A thin wire probe is immersed in the moving cuvette; a clot begins to form between the probe and the side of the cuvette. The strength of the clot, as well as a variety of other parameters, are measured over time. In rotational thromboelastometry (ROTEM), the cuvette containing blood is stationary, and the wire probe oscillates; the clot impedes movement of the probe and permits measurement of several variables [2].

\subsection{Variables Analyzed}

Variables of interest in TEG or ROTEM include maximum amplitude (MA, measured in $\mathrm{mm}$ ), a reflection of clot strength; reaction time ( $\mathrm{R}$, measured in sec), the time until there is first evidence of clot; clot kinetics $(\mathrm{K}$, measured in sec), or the time from $\mathrm{R}$ until the clot reaches $20 \mathrm{~mm}$; $\alpha$, an angular measurement from a tangent line drawn to the curve of the TEG tracing starting from the point of clot reaction time; coagulation index (CI, measured in dynes/sec), a description of a patient's global coagulable state derived from

$\mathrm{CI}=-0.2454 \mathrm{R}+0.0184 \mathrm{~K}+0.1655 \mathrm{MA}-0.0241 \alpha-5.0220$ [3].

Other parameters examined in this assay include lysis at 30 minutes (expressed as a percentage of the clot lysed) and clot firmness ( $G$, measured in dynes $/ \mathrm{cm}^{2}$ ), also called the shear elastic modulus strength [3]. From these data, a plot of MA versus time provides a graphic representation of clotting in both the coagulation phase and the fibrinolysis phase (Figure 1). The values for each TEG parameter, along with the resultant plot, can be compared to known TEG values for a variety of a blood conditions. For example, factors that will prolong $\mathrm{R}$ include decreased 


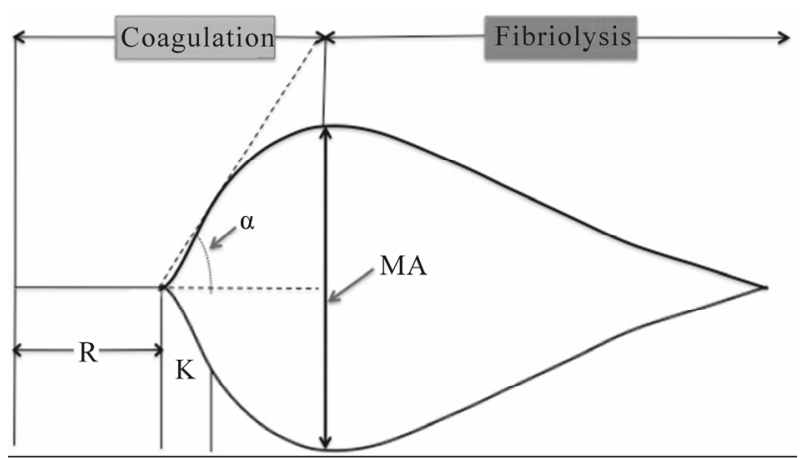

Figure 1. Normal TEG tracing, depicting rate of formation and degradation of clot, as well as the MA, R, $\alpha$ and $\mathrm{K}$.

coagulation factor production or qualitative dysfunction of coagulation factors, as well as the presence of heparin. Decreases in MA reflect platelet dysfunction or thrombocytopenia, whereas an increased MA. Conversely, increases in MA, coupled with decreased R, are suggestive of a hypercoagulable state [2].

\subsection{Sample Modifications}

Recently, modifications to the TEG sample have improved ease of sample handling and removed the necessity of performing the test promptly after sample collection. These modifications also allow specific areas of the TEG assessment to be parsed out while controlling other patient variables (Table 1). With these modifications to TEG, ex vivo analysis of various therapies can be PERFORMED USING the patient's blood to permit efficient titration to therapeutic levels. For example, by adding arachadonic acid to a sample, one can focus on the pathway affected by aspirin, thereby analyzing the dose needed to observe inhibition of the pathway and benefit the patient. Isolation of the pathway by which clopidogrel exerts its therapeutic effect is achieved by adding ADP to the sample [1].

Despite the impressive advances in TEG technology, there remain notable pitfalls to the widespread use of this test. Due to variability in the glycoprotein (GP) IIb/IIIa receptor number expressed by each person, platelet number and function, and fibrinogen concentration, a normal TEG value for one person may represent a derangement in another. Thus, a baseline TEG is an important first-step for any patient for whom TEG monitoring will be used [1]. This fact alone has enormously limited its practical use, as standardization and validation of a "normal" value has proven challenging [1,3,4].

While the advent of additive reagents and computer software implementation have greatly improved the standardization of samples before and during analysis, TEG remains a test best used to assess changes in global coagulative function over time in patients for whom a baseline is available [4].
Table 1. Modifications to TEG permit study of specific patient variables (From A. R. Hobson, R. A. Agarwala, R. A. Swallow, K. D. Dawkins and N. P. Curzen, "Thrombelastography: Current Clinical Applications and Its Potential Role in Interventional Cardiology," Platelets, Vol. 17, No. 8, 2006, pp. 509-518.)

\begin{tabular}{|c|c|}
\hline Reagent & Outcome \\
\hline Citrate & $\begin{array}{l}\text { Enables prolonged storage } \\
\text { of samples before analysis }\end{array}$ \\
\hline Heparin & $\begin{array}{c}\text { Inhibits thrombin, allowing the } \\
\text { contribution of fibrin } \\
\text { and platelets to be studied }\end{array}$ \\
\hline Heparinase & Reverses the effects of heparin \\
\hline $\begin{array}{c}\text { Activators } \\
\text { (celite, kaolin, tissue factor) }\end{array}$ & Increase speed of result acquisition \\
\hline $\begin{array}{l}\text { Glycoprotein } \\
\text { IIb/IIIa inhibitors }\end{array}$ & $\begin{array}{l}\text { Inhibits platelet function } \\
\text { allowing the contribution } \\
\text { of fibrinogen to be assessed }\end{array}$ \\
\hline $\begin{array}{l}\text { Antifibrinolytic drugs } \\
\text { (tranexamic acid) }\end{array}$ & Reverses fibrinolysis \\
\hline Reptillase and factor XIIIIa & $\begin{array}{l}\text { Activates fibrin formation } \\
\text { without affecting platelets }\end{array}$ \\
\hline Arachadonic acid & $\begin{array}{c}\text { Activates platelets via } \\
\text { production of thromboxane A2 }\end{array}$ \\
\hline $\mathrm{ADP}$ & $\begin{array}{c}\text { Activates platelets } \\
\text { via P2Y1 and P2Y12 receptors }\end{array}$ \\
\hline
\end{tabular}

\section{Current Applications}

The application and development of this method of blood analysis has evolved based heavily on the needs of the modern anesthesiologist.

\subsection{Liver Transplantation}

The liver is the major site of clotting factor production and as such, acute and chronic liver failure can contribute to a hypocoagulable state. Thrombocytopenia, due to disseminated intravascular coagulation (DIC) or hypersplenism, can compound this coagulation factor deficiency. Azotemia stemming from hepatorenal syndrome can further aggravate an existing platelet deficiency by causing qualitative platelet dysfunction [5].

Liver transplant patients represent a unique population of surgical patients, given the systemic reach of their illness and the challenges they face intraoperatively and postoperatively. During the anhepatic phase of transplantation, levels of tissue plasminogen activator (tPA) from the diseased liver are still increasing, contributing further to the existing hypocoagulable state. Results of serial TEGs drive transfusion decisions: the importance of avoiding excessive transfusion cannot be overlystressed, as these patients often experience massive blood loss but can be hypertensive in the reperfusion stage. Also during the reperfusion stage, supplementation of coagulation factors is still crucial, as the newly trans- 
planted liver resumes its synthetic capabilities [2,5]. Ultimately, the benefit of TEG in liver transplant is clear: it has been shown to reduce transfuse requirements, even in this population in which transfusion is a mainstay of intraoperative therapy, by quantifying the exact need of platelets and coagulation factors [6].

\subsection{Cardio-Pulmonary Bypass Surgery}

Extracorporeal circulation causes platelet dysfunction, coagulation factor activation and ultimately, coagulation factor depletion by passing blood through plastic or metal surfaces, permitting fibrinogen and von Willebrand Factor (vWF) adherence. Platelets can subsequently adhere to these substances, activating the coagulation cascade and causing thrombus formation [1,2,7]. Further, the procedure itself can cause clotting derangement: disruption of the endothelium exposes tissue factor, and activates the extrinsic coagulation cascade, while surgery itself induces inflammation, activating platelets [1]. As a result, cardiac surgery patients receive large doses of heparin during bypass to avoid thrombus formation [1]. The dose is so large that it exceeds the capability of aPTT to monitor coagulation in these patients; while many physicians monitor patients on cardio-pulmonary bypass (CPB) using activated clotting time (ACT), this assay provides only a fragment of the global coagulative function [1,2]. TEG permits assessment of coagulation both in the presence of heparin as well as in isolation of it, ultimately improving the specificity of blood products selected, reducing the amount of blood transfused—and even the number of patients receiving transfusion $[1,2,8]$. Moreover, TEG also provides temperature-specific information, crucial in the setting of $\mathrm{CPB}$, given that the patient's temperature is substantially cooler than normal [8].

\subsection{Obstetrics}

Up to $8 \%$ of healthy pregnant women exhibit some degree of thrombocytopenia; given the extent of screening in this population, it is not surprising that even modest, asymptomatic thrombocytopenic states are detected [9]. Though often not a cause of a marked changes in coagulation, platelet counts remain a major factor in obstetric anesthesia decisions. TEG is being more-routinely used to predict the risk of morbidity following epidural placement [1]. It can also be used to differentiate the physiologic hypercoagulable state often observed in pregnancy coagulation screening from pre-eclampsia and HELP syndrome [1]. TEG may play an important role in postpartum hemorrhage by helping clinicians rapidly identify and treat exactly the deficiency in question. This is important not only from a patient care perspective, but also as a means of preserving precious blood product resources [10].

\section{Future Directions}

\subsection{Cardiology}

The applications of TEG are ever expanding. In the area of cardiology, the use of TEG has expanded into monitoring of patients on antithrombotic therapy, such as GP IIb/IIIa antagonists or aspirin [1,11]. Given the immense variability in ADP receptor expression through the species, TEG is particularly useful in patients who appear to be "non-responders" to anti-thrombotic therapy as it permits ex vivo optimization of treatment [11,12]. The platelet mapping assay, a modification of TEG using some of the reagents in Table 1, may be useful for predicting blood loss in patients who underwent CPB [13]. This could help clinicians sooner activate massive transfusion protocols whilst helping to reduce blood product waste.

\subsection{Neonatology and Pediatrics}

The application of TEG to a specific subset of patientsneonates and infants - continues to undergo development, may be useful for predicting blood loss in patients who will undergo CPB [13]. Even in the absence of suspicion of coagulopathy, TEG can be used in neonates to detect early sepsis. Specifically, sepsis in this group is known to prolong the $\mathrm{R}$ and $\mathrm{K}$ time, while reducing the MA; early detection of these abnormalities permits intervention before the point of disseminated intravascular coagulation [14]. However, reference ranges have yet to be firmly established for this age group. The work of Oswald and colleagues sought to begin the establishment of a reference range for TEG values in the pediatric population, including neonates and infants [15]. Of particular note is the discrepancy this and other works have found between standard coagulation panel results (PT and PTT) and TEG: while standard coagulation test results suggest that neonates and infants have prolonged clotting times, TEG results suggest that these age groups have accelerated coagulation and clot firmness [15,16]. Notably, healthy newborns show no increase in bleeding tendency [15]. Given these discrepancies in results, it is not advisable to use standard coagulation panels and TEG interchangeably in neonates [16]. The poor correlation of established PT and PTT results with disorder severity can lead to an overestimation of the need for coagulation therapy [15-17]. Oswald and colleagues also found a significant difference in TEG parameters observed between the $0-3$ months group and the $4-24$ months group, suggesting that different reference ranges should be used in the assessment of these age groups. When monitored with TEG during cardiac surgery on 
$\mathrm{CPB}$, fewer neonates required plasma transfusions intraoperatively [18], a significant finding considering the increased risk of acute lung injury associated with plasma transfusion in both adult and pediatric patients. Further, TEG monitoring appeared to permit early identification of neonatal patients at-risk for post-operative mediastinal bleeding, guiding hemostatic management in this population that is particularly sensitive to volume overload [19].

\subsection{Trauma}

TEG may also have a unique role in trauma assessment. Acute traumatic coagulopathy (ATC) is a devastating consequence of traumatic injury stemming from tissue damage and systemic hypoperfusion [20,21]. Early, aggressive resuscitation with fresh frozen plasma and other blood products is paramount to these patients' survival [22]. Given its unique ability to assess whole blood, TEG is ideal for rapidly identifying patients with ATC and subsequently activating massive transfusion protocols, providing these patients with the chance of a better outcome [20]. TEG has further been used to identify the mechanism of platelet dysfunction in traumatic brain injury (TBI) that of ADP receptor inhibition [21]. While the cause for this inhibition remains unknown, early identification of this state may contribute to successful treatment of coagulopathy associated with TBI in the future [21].

\section{Conclusion}

Though TEG has been in existence for over 60 years, its potential contributions to medicine are just beginning to be realized. Now a point of care test, this whole blood method of coagulation monitoring rapidly provides a wealth of information beyond traditional, plasma-based assays. As further data become available with regard to reference values and standardization, the use of TEG is certain to become more widespread.

\section{REFERENCES}

[1] A. R. Hobson, R. A. Agarwala, R. A. Swallow, K. D. Dawkins and N. P. Curzen, "Thrombelastography: Current Clinical Applications and Its Potential Role in Interventional Cardiology,” Platelets, Vol. 17, No. 8, 2006, pp. 509-518. doi:10.1080/09537100600935259

[2] M. Kroll, "Thromboelastography: Theory and Practice in Measuring Hemostasis," Clinical Laboratory News, Vol. 36, No. 12, 2010, pp. 8-10.

[3] R. M. Edwards, B. J. Naik-Mathuria, A. N. Gay, O. O. Olutoye and J. Teruya, "Parameters of Thromboelastography in Healthy Newborns," American Journal of Clinical Pathology, Vol. 130, No. 1, 2008, pp. 99-102. doi:10.1309/LABNMY41RUD099J2
[4] Y. Dai, A. Lee, L. A. Critchley and P. F. White, "Does Thromboelastography Predict Postoperative Thromboembolic Events? A Systematic Review of the Literature,” Anesthesia \& Analgesia, Vol. 108, No. 3, 2009, pp. 734742. doi:10.1213/ane.0b013e31818f8907

[5] C. G. Krenn and A. M. De Wolf, "Current Approach to Intraoperative Monitoring in Liver Transplantation,” Current Opinion in Organ Transplantation, Vol. 13, No. 3, 2008, pp. 285-290.

doi:10.1097/MOT.0b013e3283005832

[6] R. J. McCarthy, K. J. Tuman, B. Chen and A. D. Ivankovich, "Platelet Integrin Inhibition with c7E3 Enhances the Correlation between Platelet Aggregometryand Thrombelastograph $\left.{ }^{\circledR}\left(\mathrm{TEG}^{\circledR}{ }^{\circledR}\right]\right)$ MA Values,” Anesthesia \& Analgesia, Vol. 86, 1998, p. S219. doi:10.1097/00000539-199802001-00217

[7] A. B. Gelb, R. I. Roth, J. Levin, M. J. London, R. A. Noall, W. W. Hauck, M. Cloutier, E. Verrier and D. T. Mangano, "Changes in Blood Coagulation during and Following Cardiopulmonary Bypass: Lack of Correlation with Clinical Bleeding,” Journal of Clinical Pathology, Vol. 106, No. 1, 1996, pp. 87-99.

[8] L. Shore-Lesserson, H. E. Manspeizer, M. DePerio, S. Francis, F. Vela-Cantos and M. A. Ergin, "Thromboelastography-Guided Transfusion Algorithm Reduces Transfusions in Complex Cardiac Surgery,” Anesthesia \& Analgesia, Vol. 88, No. 2, 1999, pp. 312-319. doi:10.1097/00000539-199902000-00016

[9] R. A. Kadir and C. McLintock, "Thrombocytopenia and Disorders of Platelet Function in Pregnancy,” Seminars in Thrombosis and Hemostasis, Vol. 37, No. 6, 2011, pp. 640-652. doi:10.1055/s-0031-1291374

[10] O. Onwuemene, D. Green and L. Keith, "Postpartum Hemorrhage Management in 2012: Predicting the Future," International Journal of Gynecology \& Obstetrics, Vol. 119, No. 1, 2012, pp. 3-5. doi:10.1016/j.ijgo.2012.07.001

[11] J. H. Waters, D. G. Anthony, A. Gottlieb and J. Sprung, "Bleeding in a Patient Receiving Platelet Aggregation Inhibitors,” Anesthesia \& Analgesia, Vol. 93, No. 4, 2001, pp. 878-882. doi:10.1097/00000539-200110000-00015

[12] L. Bochsen, B. Wiinberg, M. Kjelgaard-Hansen, D. A. Steinbrüchel and P. I. Johansson, "Evaluation of the TEG Platelet Mapping Assay in Blood Donors," Thrombosis Journal, Vol. 5, 2007, p. 3. doi:10.1186/1477-9560-5-3

[13] N. S. Weitzel, L. B. Weitzel, L. E. Epperson, A. Karimpour-Ford, Z. V. Tran and T. Seres, "Platelet Mapping as Part of Modified Thromboelastography (TEG) in Patients Undergoing Cardiac Surgery and Cardiopulmonary Bypass,” Anesthesia \& Pain Management, Vol. 67, No. 10, 2012, pp. 1158-1165. doi:10.1111/j.1365-2044.2012.07231.x

[14] H. W. Grant and G. P. Hadley, "Prediction of Neonatal Sepsis by Thromboelastography,” Pediatric Surgery International, Vol. 12, No. 4, 1997, pp. 289-292. doi:10.1007/BF01372152

[15] E. Oswald, B. Stalzer, E. Heitz, M. Weiss, M. Schmugge, A. Strasak, P. Innerhofer and T. Haas, "Thromboelastography (ROTEM) in Children: Age-Related Reference Ranges and Correlations with Standard Coagulation Tests," 
British Journal of Anaesthesia, Vol. 105, No. 6, 2010, pp. 827-835. doi:10.1093/bja/aeq258

[16] T. Haas, N. Spielmann, J. Mauch, C. Madjdpour, O. Speer, M. Schmugge and M. Weiss, "Comparison of Thromboelastography (ROTEM ${ }^{\circledR}$ ) with Standard Plasmatic Coagulation Testing in Paediatric Surgery,” British Journal of Anaesthesia, Vol. 108, No. 1, 2012, pp. 36-41. doi:10.1093/bja/aer342

[17] M. Radicioni, D. Mezzetti, A. Del Vecchio and M. Motta, "Thromboelastography: Might Work in Neonatology Too?” Journal of Maternal-Fetal and Neonatal Medicine, Vol. 25, No. S4, 2012, pp. 10-13. doi:10.3109/14767058.2012.714996

[18] B. S. Romlin, H. Wahlander, H. Berggren, M. Synnergren, F. Baghaei, K. Nilsson and A. Jeppsson, "Intraoperative Thromboelastography Is Associated with Reduced Transfusion Prevalence in Pediatric Cardiac Surgery,” Anesthesia \& Analgesia, Vol. 112, No. 1, 2011, pp. 30-36. doi:10.1213/ANE.0b013e3181fe4674

[19] S. Moganasundram, B. J. Hunt, K. Sykes, F. Holton, K. Parmar, A. Durward, I. A. Murdoch, C. Austin, D. Ander- son and S. M. Tibby, “The Relationship among Thromboelastography, Hemostatic Variables, and Bleeding after Cardiopulmonary Bypass Surgery in Children,” Anesthesia \& Analgesia, Vol. 110, No. 4, 2010, pp. 995-1002.

[20] R. Davenport and S. Khan, "Management of Major Trauma Haemorrhage: Treatment Priorities and Controversies," British Journal of Anaesthesia, Vol. 155, No. 5, 2011, pp. 537-548. doi:10.1111/j.1365-2141.2011.08885.x

[21] P. K. Davis, H. Musunuru, M. Walsh, R. Cassady, R. Yount, A. Losiniecki, E. E. Moore, M. V. Wohlauer, J. Howard, V. A. Ploplis, F. J. Castellino and S. G. Thomas, "Platelet Dysfunction Is an Early Marker for Traumatic Brain Injury-Induced Coagulopathy,” Neurocritical Care, 2012. doi:10.1007/s12028-012-9745-6

[22] C. Ives, K. Inaba, B. C. Branco, O. Okoye, H. Schochl, P. Talving, L. Lam, I. Shulman, J. Nelson and D. Demetriades, "Hyperfibrinolysis Elicited via Thromboelastography Predicts Mortality in Trauma," Journal of the American College of Surgeons, Vol. 215, No. 4, 2012, pp. 496-502. doi:10.1016/j.jamcollsurg.2012.06.005 